\title{
Canadian Pandemic Influenza Preparedness: Health sector planning guidance
}

\author{
B Henry ${ }^{1,2}$ on behalf of the Canadian Pandemic Influenza Preparedness (CPIP) Task Group*
}

\begin{abstract}
Pandemic preparedness requires a multifaceted approach with collaboration from all levels of government. The Canadian Pandemic Influenza Preparedness: Planning Guidance for the Health Sector (CPIP) is a guidance document that outlines key health sector preparedness activities designed to ensure Canada is ready to respond to the next influenza pandemic. This article outlines Canada's approach to pandemic influenza preparedness as described in the CPIP Main Body. Canada's pandemic influenza preparedness planning takes place within a network of legislated requirements and emergency frameworks at provincial/territorial, federal and international levels. The plan includes several guiding principles, including collaboration among governments and stakeholders, evidence-based decision-making, proportionality and flexibility in tailoring responses to the situation, the adoption of a precautionary approach, the use of established practices and systems and the explicit incorporation of ethical principles in all decisions and decision-making processes. The roles and responsibilities of the federal and provincial/territorial governments is identified and three planning tools are provided: planning assumptions rooted in evidence; multiple scenarios to support decision-making; and descriptive terms such as the start, peak and end of pandemic wave rather than phase terminology to provide triggers for action. Overall, the CPIP Main Body sets out a scalable, coordinated risk management approach to an influenza pandemic. This is an evergreen document that will be updated regularly.
\end{abstract}

\author{
Affiliations \\ ${ }^{1}$ CPIP Task Group Chair \\ 2 Office of the Provincial Health \\ Officer, Victoria, BC

*Correspondence:
CPIPTGSecretariat-
GTPCPSecretariat@phac-aspc.
gc.ca

Suggested citation: Henry B on behalf of the Canadian Pandemic Influenza Preparedness (CPIP) Task Group, Canadian Pandemic Influenza Preparedness: Health sector planning guidance. Can Commun Dis Rep.

2018;44(1):6-9. https://doi.org/10.14745/ccdr.v44i01a02

\section{Introduction}

Influenza pandemics are infrequent occurrences that emerge when a novel influenza A virus with sustained human-to-human transmission causes widespread human illness. Governments must make advance preparations to respond to an influenza pandemic, as it is impossible to predict when a pandemic may occur, or how severe it will be.

This article summarizes the main body of Canada's pandemic influenza planning approach, as set out in the Canadian Pandemic Influenza Preparedness (CPIP): Planning Guidance for the Health Sector (1). The CPIP provides guidance to the federal, provincial and territorial (FPT) jurisdictions that are responsible for preparing for and responding to an influenza pandemic, and is aimed primarily at FPT ministries of health and other ministries that have health responsibilities. It is not a pandemic response plan in itself.

Effective collaboration among all FPT governments is necessary in the planning and delivery of response activities. Accordingly, the CPIP describes how the FPT "jurisdictions will work together to ensure a coordinated and consistent health sector approach to pandemic preparedness and response" (1). The strategy and guidance described in the CPIP main body were approved by the Pan-Canadian Public Health Network (PHN) Council (2) and the Conference of FPT Deputy Ministers of Health, and it is anticipated that FPT planning will align with the strategic direction of the CPIP.

Since the CPIP is built as much as possible on existing health sector functions and structures, such as surveillance and control measures, it supports all-hazards response plans that apply to any type of public health emergency.

\section{The updated plan}

The CPIP provides planning guidance for the health sector for pan-Canadian influenza preparedness and response. It is intended to minimize illness and overall deaths, and to minimize societal disruption from an influenza pandemic.

New aspects of the CPIP include:

- guiding principles and approaches, such as the consideration of ethics and Canada's diversity,

- the adoption of a risk management approach, with updated planning assumptions, and

- $\quad$ planning tools to assist provinces/territories in developing their own plans. 
Some elements in the updated CPIP reflect lessons that were learned in the $2009 \mathrm{H} 1 \mathrm{~N} 1$ influenza pandemic (3). For example, due to the high demand for some response elements, such as surveillance activities and critical care medical equipment, it was recommended that surveillance systems and epidemiology capacity and links with primary care providers, be strengthened. As a result of the variation in timing and intensity of pandemic waves, greater scalability and adaptability of response measures have been incorporated, with a set of triggers for action that identify the pandemic conditions at which certain responses should be activated and deactivated.

The main body describes the background and rationale for pandemic influenza preparedness planning in general and for the approach taken in the Canadian context in particular. This broad strategic guidance is complemented by a set of technical annexes that provide more detailed guidance and advice specific to many of the key functional elements of pandemic preparedness and response, while also incorporating the broader strategic principles of the main body. Annexes for three key response functions, surveillance, laboratory services and vaccines, have recently been updated to reflect lessons learned from the $2009 \mathrm{H} 1 \mathrm{~N} 1$ pandemic.

This article is the second in a series; providing an update on the different sections of the CPIP. Additional articles on the laboratory strategy and surveillance strategy annexes follow later in this issue of the CCDR $(4,5)$. The first article in the series was a summary of the CPIP's Vaccine Annex (6).

\section{Context for planning}

\section{Legislation and emergency frameworks}

Canada's pandemic influenza preparedness planning takes place within a network of legislated requirements and emergency frameworks at provincial/territorial, federal and international levels. The federal government's preparedness plans for public health emergencies are part of the broader emergency management system that is managed by Public Safety Canada (7), and there is also a system of FPT health emergency plans. Canada also collaborates with several international partners to ensure regional preparedness for an influenza pandemic under agreements such as the Pandemic Influenza Preparedness Framework (World Health Assembly) (8) and the North American Plan for Animal and Pandemic Influenza (NAPAPI) (9) with Mexico and the United States.

\section{Other planning considerations}

The updated CPIP was prepared with consideration of the diversity of Canada, which reflects the geographic size and variability of the country, and the ethnic, language, religious, cultural and lifestyle diversity of the population. Examples of planning considerations include the presence of many small, remote and isolated communities across the country that are less well served by health and other services and the many individuals and groups who are more vulnerable to health emergencies; for example, those who are physically or mentally disabled, are low income or are homeless.
These factors are of particular relevance to pandemic preparedness and response, primarily through the need to support a response that is flexible to local conditions and to the needs of specific or vulnerable people. Ethical principles are explicit in the updated CPIP; guiding decisions that are based on the health and interests of a population rather than on clinical ethics that are based on the interests of individuals. This orientation implies a need to encourage a sense of solidarity within a community and reciprocity with those who may require greater support.

\section{Guiding principles}

The updated CPIP is underpinned by a set of guiding principles. These include collaboration among governments and stakeholders, evidence-based decision-making and proportionality and flexibility in tailoring responses to the situation. Three more general approaches are also applied: in the adoption of a precautionary or protective approach, particularly in the early stages when uncertainties are high; the use of established practices and systems, rather than attempting to adopt new approaches during an emergency; and the explicit incorporation of ethical principles in all decisions and decision-making processes.

\section{Guidance for preparedness and response}

\section{Coordination of roles and responsibilities}

Preparedness and response to an influenza pandemic require a whole-of-government approach to ensure the commitment of all necessary resources to minimize health, societal and economic impacts, and these contributions must be coordinated. The health sector pandemic preparedness activities that are described in the CPIP require the participation of international and FPT levels of government; furthermore, many operational functions are carried out by a range of professional disciplines within and beyond the health sector, such as health practitioners, international regulators, vaccine manufacturers and non-governmental organizations. The delineation of the responsibilities of the FPT governments for these functions, and the mechanisms for their collaboration, are major aspects of preparedness described in the CPIP.

Internationally, the World Health Organization (WHO) conducts global risk assessments, makes the declaration of a public health emergency of international concern, selects the pandemic vaccine strain and determines the switch from seasonal to pandemic vaccine production. Liaison with this and other international organizations in pandemic management is a federal government responsibility.

The coordination of a pan-Canadian response requires collective infrastructure and coordinated activities; for example, the federal government is responsible for the regulatory aspects of testing and approvals for influenza vaccines and antiviral medications, for negotiating with manufacturers and establishing contracts for the FPT purchase of influenza vaccines and antiviral medications, and for maintenance and mobilization of medical supplies in the National Emergency Strategic Stockpile (NESS) and by 
facilitating the acquisition of additional supplies (10). The PTs are responsible for the purchasing, distribution and administration of vaccines and antiviral medications within their jurisdictions.

\section{Risk management approach}

The updated CPIP introduces a risk management approach to decision-making to manage the uncertainties that are inherent in preparedness planning for pandemic influenza. Risk management is a systematic approach to setting the best course of action in an uncertain environment by identifying, assessing, acting on and communicating risks. This approach is supported by the CPIP principles of evidence-based decision-making, proportionality and flexibility, and a precautionary/protective approach in uncertain conditions.

\section{Tools for pandemic preparedness planning}

Given the large number of variables that are involved in influenza pandemic planning, comprehensive risk management is challenging. The updated CPIP contains three broad planning tools: planning assumptions; pandemic planning scenarios; and planning phases and triggers for action.

Planning assumptions are hypothetical assumptions rooted in evidence, which serve as a guide to manage uncertainty and provide a useful framework for planning phases. As a pandemic unfolds, emerging evidence will replace the assumptions and be used to guide the response.

To help with risk identification, multiple scenarios have been defined to support planning and evidence-informed decision-making. Planning scenarios provide a starting point to think through implications and risks that would be associated with pandemics of varying population impacts, from low to high.

Descriptive terms for planning phases, such as the start, peak and end of a pandemic wave, are defined in the CPIP. Previously, the WHO's phase terminology (interpandemic, alert, pandemic, transition) was used to describe pandemic activity in the country or in a jurisdiction within Canada. Triggers for action provide guidance for initiation of FPT activities and for their modification and cessation. Pandemic response should be appropriate to the local situation to ensure PT, or regional/local level response is appropriate to the situation.

\section{Assessment and evaluation}

Preparing for and responding to a pandemic is a complex process that requires the coordinated efforts of all levels of government in collaboration with stakeholders. To ensure pandemic plans (or all-hazards plans, according to the jurisdiction) are comprehensive and effective, jurisdictions should assess their level of preparedness, test their plans regularly, and evaluate their pandemic response.

\section{Discussion}

The updated CPIP responds to several challenges that are inherent in planning influenza pandemic preparedness and response in Canada, which include not only the uncertainties that are inherent in influenza pandemics but also the scale, diversity and jurisdictional divisions in Canada.

The CPIP addresses the uncertainties of pandemic influenza, through a risk management approach that is scalable to different pandemic impact levels (low, moderate and high) and to changing impacts throughout the progress of a pandemic. This approach also provides the flexibility that is needed for decision makers to tailor a response to the needs and capacities of different regions in Canada, adjusting to regional variations in timing and intensity of pandemic impact, as well as to diverse communities and populations.

While flexibility is needed to allow different jurisdictions to tailor their plans and pandemic response activities to regional needs and conditions, shared objectives and a consistent approach are also needed to enable the jurisdictions to collaborate on delivering response activities. To provide consistency in the approach to pandemic planning among FPT jurisdictions and to aid in collaboration among response partners, the CPIP articulates a set of principles and a consideration of ethics and of the diverse and vulnerable populations in Canada with which pandemic plans must align. More operationally, collaboration among jurisdictions and between jurisdictional levels is critical to the effective response to a pandemic. The CPIP provide a delineation of roles and responsibilities for preparedness and response activities nationally, and define a process for interaction and communication between and among jurisdictions. These collaborative roles, structures and processes form a major part of pandemic preparedness in Canada.

The broad principles and considerations, risk management approach and structures and processes for collaboration that are set out in the CPIP are carried through into the more detailed guidance that has been developed for the response components.

\section{Conclusion}

The CPIP is an evergreen document that will be updated regularly with new information, legislative changes/agreements or best practices as required. A more comprehensive and fulsome review of the CPIP and its technical annexes will occur every five years to ensure the document is up-to-date and meeting the needs of FPT governments, health professionals and stakeholders. The Main Body will undergo its next full review in 2019.

\section{Authors' statement}

Canadian Pandemic Influenza Preparedness Task Group (CPIPTG): Henry B (Chair), Alfieri A, Gant S, Gemmill I, Hatchette T, Jayaraman $G$ and Schwartz B

CPIPTG Secretariat: Paddle L, Stirling R and Gadient S

PHAC: Charos G, Williams J

\section{Conflict of interest}

None. 


\section{Acknowledgements}

Many thanks to Anne Wiles who prepared the initial draft of this summary.

\section{Funding}

The work of the Canadian Pandemic Influenza Preparedness Task Group is supported by the Public Health Agency of Canada.

\section{References}

1. Public Health Agency of Canada. Canadian Pandemic Influenza Preparedness: Planning Guidance for the Health Sector. Ottawa (ON): PHAC; 2017. https://www.canada.ca/ en/public-health/services/flu-influenza/canadian-pandemicinfluenza-preparedness-planning-guidance-health-sector. html

2. Pan-Canadian Public Health Network Council. http://www. phn-rsp.ca/index-eng.php

3. Public Health Agency of Canada. Lessons Learned Review: Public Health Agency of Canada and Health Canada Response to the 2009 H1N1 Pandemic. Ottawa (ON): PHAC; 2010. http://www.phac-aspc.gc.ca/about_apropos/ evaluation/reports-rapports/2010-2011/h1n1/index-eng.php

4. Henry B on behalf of the Canadian Pandemic Influenza Preparedness Task Group. Canada's pandemic Influenza preparedness: laboratory strategy. Can Commun Dis Rep 2018;44(1):10-3. https://www.canada.ca/en/public-health/ services/reports-publications/canada-communicable-disease- report-ccdr/monthly-issue/2018-44/ccdr-volume-44-1january-4-2018/canadas-pandemic-laboratory-strategy.html

5. Henry B on behalf of the Canadian Pandemic Influenza Preparedness Task Group. Canada's pandemic influenza preparedness: surveillance strategy. Can Commun Dis Rep. 2018;44(1):14-7. https://www.canada.ca/en/publichealth/services/reports-publications/canada-communicabledisease-report-ccdr/monthly-issue/2018-44/ccdr-volume-441-january-4-2018/canadas-pandemic-surveillance-strategy. html

6. Henry B. Gadient S on behalf of the Canadian Pandemic Influenza Preparedness Task Group. Canada's pandemic vaccine strategy. Can Commun Dis Rep. 2017;43(7/8):160-3. https://www.canada.ca/content/dam/phac-aspc/migration/ phac-aspc/publicat/ccdr-rmtc/17vol43/dr-rm43-7-8/assets/ pdf/17vol43_7_8-ar-05-eng.pdf

7. Public Safety Canada. Emergency Management. 2016. https://www.publicsafety.gc.ca/cnt/mrgnc-mngmnt/index-en. aspx

8. World Health Organization. World Health Assembly Pandemic Influenza Preparedness Framework. 2011 World Health Organization. http://www.who.int/influenza/pip/en/

9. North American Plan for Animal and Pandemic Influenza (NAPAPI). Ottawa (ON): Public Safety Canada; 2016. https:// www.publicsafety.gc.ca/cnt/rsrcs/pblctns/nml-pndmc-nflnz/ index-en.aspx

10. Public Health Agency of Canada. National Emergency Strategic Stockpile. Ottawa (ON): PHAC; 2015. https:// www.canada.ca/en/public-health/services/emergencypreparedness-response/national-emergency-strategicstockpile.html 